\title{
Zur Differentialdiagnose zwischen Dementia paralytica und Lues des Zentralnervensystems.
}

\author{
Von \\ Axel Bisgaard. \\ (Aus der Kopenhagener Irrenanstalt „St.-Hans-Hospital“ [Direktor: Professor \\ Dr, O. Rohmell].)
}

(Eingegangen am 18. Dezember 1911.)

In einer früheren Arbeit (E. Bertelsen und A. Bisgaard, diese Zeitschr. Orig. 4, 327. 1911) habe ich, in der Absicht, qualitative Bestimmungen über die Art der Fiweißstoffe auszuführen (1. c. S. 350 Tab. 5), mit Ammoniumsulfat in halbgesättigter Lösung einige Fraktionierungsversuche mit Spinalflüssigkeit gemacht. Es zeigte sich daß die Hauptmenge der Eiweiße - namentlich bei den Paralysen (aber auch in drei anderen Fällen) - mit Ammoniumsulfat gefällt wurde (,Globuline"). Unter den sogenannten Paralysen befand sich aber ein Fall (Nr. 87), der einen beträchtlichen Gehalt an „Albuminen" (d. s. Proteinstoffe, die mit Ammoniumsulfat in halbgesättigter Lösung keinen Niederschlag geben) zeigte.

Bei der Fortsetzung dieser Versuche bin ich vorläufig zu folgendem Resultate gekommen:

Findet sich in einer Cerebrospinalflüssigkeit eine beträchtliche Vermehrung des Eiweißes, so ist diese regelmäßig durch eine organische Krankheit des Zentralnervensystems verursacht. Bei Dementia paralytica wird die Hau ptmenge des Eiweißes mit Ammoniumsulfat gefällt, bei allen übrigen organischen Krankheiten, die ich untersucht habe, darunter auch Lues cerebri, wird dagegen mit Ammoniumsulfat nur ein kleinerer Bruchteil des Eiweißes gefällt.

Ich habe im ganzen 47 Fälle, nämlich 27 Paralysen, 1 Tabes dorsalis, 1 manisch-depressive Psychose, 1 Delirium acutum, 1 Delirium tremens protrah., 5 Lues cerebri, 4 Tumor cerebri (nicht luetisch) und 7 akute und subakute durch verschiedene andere Ursachen hervorgerufene Meningitiden untersucht. In weitaus der Mehrzahl der Fälle ist natürlich die Eiweißmenge vermehrt gewesen. Diese habe ich auf meine gewöhnliche Weise (Monatsschr. f. Psych. u. Neurol. 28, 125) so ge- 
messen, daß von den zwei die Verdünnungstiter angebenden Zahlen die erste die Zahl für Ammoniumsulfat, die zweite für Salpetersäure anzeigt, während angenommen wird, daß die normale sich von 0 bis 2 und von 6 bis 15 (früher angegeben: 20) bewegt. Die Technik ist im übrigen die folgende gewesen. Erst wird die Eiweißmenge in gewöhnlicher Weise bestimmt; dann versetze ich 1-2 ccm der Spinalflüssigkeit mit einem gleichen Volumen gesättigter reiner Ammoniumsulfatlösung und schüttle kräftig. Der gebildete Niederschlag senkt sich im Laufe von ca. 12 Stunden so weit zu Boden, daß die obenstehende klare Flüssigkeit vorsichtig abpipettiert werden kann (Zentrifugierung gibt merkwürdigerweise trotz den Angaben Szecsis nur ausnahmsweise klare Flüssigkeit, selbst wenn sie mehrere Stunden hindurch kräftig ausgeführt wird). Die abpipettierte Fraktion wird aufs neue der Probe mit Salpetersäure mit Verdünnungen auf gewöhnliche Weise unterworfen.

Man bekommt dann folgende Resultate, für welche ich als Beispiel erstens 5 Paralysen anführe:

$$
\begin{array}{ll}
\text { Nr. } 55\left\{\begin{array}{cl}
4-15 \\
2-8
\end{array}\right. & \text { Nr. } 93\left\{\begin{array}{l}
6-20 \\
2-8
\end{array}\right.
\end{array} \quad \text { Nr. } 49\left\{\begin{array}{l}
4-30 \\
2-15
\end{array}\right.
$$

Die oberen zwei Ziffern sind die resp. Reaktionen mit Ammoniumsulfat und Salpetersäure vor der Fällung mit Ammoniumsulfat, die unteren Ziffern dieselben Reaktionen im Filtrate nach der Fällung. (Die Ziffer ,2" für Ammoniumsulfat ist natürlich hier konstant und bedeutet insofern nichts. Eine halbgesättigte Lösung des Ammoniumsulfats gibt ja mit einer gesättigten Lösung desselben Salzes eine ganz neue Art von Eiweißfällung (vgl. Hof meister und seine Schüler).) Man sieht also die Fällung, ganz wie ich früher (l. c. S. 350) in der angeführten Tab. 5 gezeigt habe, trotz den ansteigenden gesammelten Eiweißmengen, bis zu einem gewissen niederen Niveau von Resteiweiß vor sich gehen. Nachfolgende Fälle-geben ganz andere Resultate:

$\begin{array}{ll}\text { Nr. } 147 \text { (Leptomeningitis acuta purulenta) } & \left\{\begin{array}{r}15-230 \\ 2-140\end{array}\right. \\ \text { Nr. } 208 \text { (Meningitis tuberculosa) } & \left\{\begin{array}{l}5-42,5 \\ 2-37,5 \\ 8-120 \\ 2-47,5\end{array}\right. \\ \text { Nr. 205 (Tumor cerebri) }\end{array}$

Die 5 Fälle von Lues cerebri gaben folgendes:
Nr. $87\left\{\begin{array}{r}20-250 \\ 2-100\end{array}\right.$
Nr. $105\left\{\begin{array}{l}8-80 \\ 2-30\end{array}\right.$
Nr. $112\left\{\begin{array}{c}10-140 \\ 2-80\end{array}\right.$
Nr. $195\left\{\begin{array}{c}15-120 \\ 2-55\end{array}\right.$
Nr. $210\left\{\begin{array}{c}15-170 \\ 2-85\end{array}\right.$ 
Wie man sieht, bleibt hier ein bedeutender Eiweißrest, der sich nicht fällen läßt, zurück. Der oben angeführte Fall der Tab. 5 (l. c.), Nr. 87, kehrt also hier als eine Nichtparalyse wieder. - Hiermit verhält es sich folgendermaßen. Der Patient hatte eine stark positive Wassermannreaktion sowohl im Blute als in der Spinalflüssigkeit. Im übrigen spricht so gut wie alles dagegen, daß er an einer Paralyse leidet. Seine an Häufigkeit zunehmenden epileptiformen Krampfanfälle mit Zungenbiß, die er vor einem halben Jahre hatte, scheinen durch eine kombinierte Hg-KI-Kur zum Aufhören gebracht worden zu sein und die Diagnose muß als ungeklärt gelten. Nr. $112 \mathrm{kam}$ zur Sektion. Es war eine Meningo-Encephalitis luetica (mikrosk. Diagnose) mit Hämorrhagien. Nr. 105 erholte sich nach einer Quecksilberkur wieder und wurde vor einem Jahre geheilt entlassen. Nr. 195 und 210 waren Wassermann-positiv im Blute, Wassermann-negativ in der Spinalflüssigkeit, Nr. 105 negativ in beiden, Nr. 112 war nicht untersucht worden, weil die Diagnose erst nach dem Tode gestellt wurde. Die Paralysen haben die gewöhnlichen Wassermannreaktionen gegeben, waren meistens im Blute positiv, die Mehrzahl zugleich auch in der Spinalflüssigkeit.

U. Friedemann (Zeitschr. f. Hygiene 1910) soll einen Antagonismus zwischen der Globulin- und der Albuminfraktion des Normalserums gezeigt haben, weil dieGlobulinfraktion komplementbindend ist, während die Albuminfraktion, wenn sie auch zugesetzt wird, die genannten Eigenschaften bei der Globulinfraktion wieder aufhebt. Bei syphilitischen Seren soll indessen die Albuminfraktion ein solches Vermögen nicht besitzen.

Trotzdem ist es von nicht geringem Interesse, daß die Paralysen, die in der Spinalflüssigkeit überwiegend Wassermann-positive sind, hier so ,globulinstark“, während die der Regel nach Wassermannnegativen Lues-cerebri-Fälle so „,albuminstark" sind. Vielleicht könnte dieses Albumin auch als überschüssiges Komplement wirken und dadurch die Hemmung unmöglich machen. Jedenfalls werden ja nicht immer Liquores bei der Wassermannschen Reaktion inaktiviert. Bei Nr. 87 war vom Eiweiße mit Ammoniumsulfat 60 Prozent fällbar (nach Gewichtsprozenten wahrscheinlich noch mehr). Jedenfalls ist es möglich, daß diese überwiegenden ,Globulin"mengen eine Rolle bei der positiven Reaktion spielen könnten. Dieses sollte dann aber auch für Nr. 105 gelten, wo es aber nicht der Fall ist.

Weil und Kafka (Med. Klinik 1911, S. 1314) finden bei Paralysen in der Spinalflüssigkeit hämolytische Amboceptoren, aber kein Komplement; bei akuten Meningitiden, und ebenso luetischen, sowohl Amboceptoren wie Komplement. Sie meinen hiermit neue Belege für die Sonderstellung der Paralyse gefunden zu haben und stellen eine interessante neue Paralysetheorie, die auf einer spe- 
zifischen universellen Gefäßerkrankung von vieljähriger Dauer gegründet sei, aut.

Meine in diesem Aufsatze niedergelegten Untersuchungen zeigen auf anderem Wege einen für die Paralyse eigentümlichen Stoffwechsel.

Nachdem Steenberg (1860), später Jespersen (1874) und Rohmell (1884) die Meinung vertreten hatten, daß die Syphilis eine „,conditio sine qua non" für die Paralyse bedeute, wurden (1896 u. folgende Jahre) die interessanten P. Heibergschen Kurven veröffentlicht, welche die Abhängigkeit zwischen Syphilis und Paralyse mit dem eigentümlichen 15jährigen Zwischenraum zwischen der Infektion und dem Tode zeigten. Wurde die Dauer der Paralyse zu ca. 3 Jahren gerechnet, sollten die ersten klinischen Symptome ca. 12 Jahre nach der Ansteckung auftreten, was auch im großen ganzen stimmte. Die Frage „Syphilis“" war mit der Wassermannreaktion endgültig entschieden. Meines Erachtens ist nur eine Erklärung der Resultate Heibergs möglich, nämlich, daß der von der Syphilis verursachte krankhafte Vorgang, der zur Paralyse führt (sei dieser nun, wie er wolle), seinen Anfang gleich im sekundären Stadium nehmen muß. Die angegebene Gesetzmäßigkeit ist ganz einfach ein Ausdruck dafür, daß ein von der Syphilis verursachtes ,Etwas“, daß durchschnittlich 12-15 Jahre zu seiner Vollbringung erfordert, vor sich geht. Die Aufgabe ist also nur, durch Frühsymptome ausfindig zu machen, was dieses „Etwas“ ist. In der Meinung, daß es sich dabei um einen chronischen meningitischen Prozeß irgend welcher Art handle, habe ich mit Boas zusammen mit der von mir beschriebenen Formolreaktion einige Zeit hindurch gesucht, krankhafte Veränderungen der Spinalflüssigkeit bei den Syphilitikern mit sekundären Efflorescenzen zu entdecken und weiter zu verfolgen. Leider aber zeigte sich die besprochene Reaktion, wäre sie auch theoretisch interessant, klinisch unbrauchbar, wenn von einem besonders ausschlaggebenden Syphilisdiagnosticum für organische Krankheiten des Zentralnervensystems die Rede war.

Weil und Kafka meinen dagegen, es handle sich um eine universelle syphilitische Gefäßerkrankung. Indem durch eine solche die Gefäße für neue Stoffe vom Blute durchdringbar werden, sollten solche immer in das Nervengewebe hinübergehen können, wo sie dann durch eine toxische Wirkung Jahre hindurch den bekannten Zerstörungsprozeß vollbringen. Ebenso in den übrigen Organen (,Kachexie"). Weil und Kafka meinen denn auch schon in früheren Stadien durch die Hämolysinreaktion eine vermehrte Gefäßpermeabilität festgestellt zu haben. Nur wird vorläufig keine Erklärung darüber gegeben, wie die Gefäßerkrankung, die zur Paralyse führen sollte, beschaffen sein mag. Biologisch und chemisch lassen sich also jedenfalls in der Spinalflüssigkeit bei der Paralyse vorläufig andere Stoffe als bei den 
gewöhnlichen luischen Erkrankungen des Zentralnervensystems, wo auch die Gefäße angegriffen sind, nachweisen.

Zum Schlusse spreche ich für t'berlassung des Krankenmaterials dem Herm Direktor Professor Rohmell und den Herren Chefärzten der betreffenden Hospitalsabteilungen und der Kliniken meinen besten Dank aus. 